Homology, Homotopy and Applications, vol.10(2), 2008, pp.115-133

\title{
PRODUCTS IN HOPF-CYCLIC COHOMOLOGY
}

\author{
ATABEY KAYGUN \\ (communicated by J. F. Jardine)
}

\begin{abstract}
We construct several pairings in Hopf-cyclic cohomology of (co)module (co)algebras with arbitrary coefficients. As a special case of one of these pairings, we recover the Connes-Moscovici characteristic map in Hopf-cyclic cohomology. We also prove that this particular pairing, along with a few others, would stay the same if we replace the derived category of (co)cyclic modules with the homotopy category of (special) towers of super complexes, or the derived category of mixed complexes.
\end{abstract}

\section{Introduction}

Hopf-cyclic cohomology of Hopf algebras was discovered by Connes and Moscovici in their seminal work on transverse index theory $[\mathbf{3}, \mathbf{4}]$. The Hopf-cyclic cohomology was later extended by Hajac, Khakhali, Rangipour and Sommerhäuser [9] to a cohomology theory of (co)module (co)algebras with coefficients in stable anti-YetterDrinfeld (SAYD) modules. Now, Hopf-cyclic cohomology of module (co)algebras as defined in $[\mathbf{9}]$ and its subsequent generalization to arbitrary coefficients in [15], can be described via appropriate bivariant cohomology theories [16]. These bivariant cohomology theories provide us with the right kind of tools to investigate various kinds of products and pairings in the Hopf-cyclic and equivariant cyclic settings. We showed in [16] that equivariant cyclic (co)homology groups of $H$-module (co)algebras are graded modules over the graded algebra $\operatorname{Ext}_{H}^{*}(k, k)$, where $H$ is the underlying Hopf algebra. Not only is this the right kind of equivariance for an equivariant cohomology theory, but it is also a very useful computational tool in obtaining new equivariant cyclic classes from old ones by using the action. However, Hopf-cyclic cohomology is, by design, oblivious to the cohomology of the underlying Hopf algebra viewed as an algebra or a coalgebra depending on the type of the symmetry at hand. Nevertheless, in Theorem 2.8 we construct a pairing similar to one of the cup products obtained in $[\mathbf{1 7}]$

$$
\smile: H C_{\mathrm{Hopf}}^{p}(C, M) \otimes H C_{\mathrm{Hopf}}^{q}(A, M) \rightarrow H C^{p+q}(A)
$$

for an $H$-module coalgebra $C$ acting equivariantly on a $H$-module algebra $A$ (Definition 2.2), where $M$ is an arbitrary coefficient module/comodule. The main corollary to this pairing is Theorem 2.10, where we recover the Connes-Moscovici

Received October 22, 2007, revised May 1, 2008; published on August 15, 2008. 2000 Mathematics Subject Classification: 16E40, 16W30.

Key words and phrases: cup product, Hopf-cyclic cohomology, characteristic map.

This article is available at http://intlpress.com/HHA/v10/n2/a5

Copyright (c) 2008, International Press. Permission to copy for private use granted. 
characteristic map [3, Section VIII, Proposition 1] by letting $q=0, C=H$, and letting $M=k_{(\sigma, \delta)}$ be the coefficient module coming from a modular pair in involution. The ideas instrumental in constructing this pairing are the derived functor interpretation of Hopf-cyclic and equivariant cyclic cohomology [16], and the Yoneda interpretation of Ext-groups [23]. Once we couple equivariant/Hopf-cyclic cohomology and the Yoneda product, we obtain other types of products and pairings by imposing various conditions on the underlying Hopf algebra, the coefficient modules and (co)module (co)algebras involved (Theorems 2.11, 3.2, 3.5, 4.4 and 4.7.)

The first example of the pairing of the form given in Theorem 2.8 between Hopfcyclic cohomology of a module algebra $A$ and the comodule algebra $C=H$ is the Connes-Moscovici characteristic map [3] defined for $q=0$, and for the 1-dimensional coefficient module $k_{(\sigma, \delta)}$ coming from a modular pair in involution. Gorokhovsky later extended this pairing to a differential graded setting using periodic cyclic cohomology for an arbitrary $q$ in [8]. Subsequently, in [17], Khalkhali and Rangipour defined two cup products for arbitrary module algebras and coalgebras with coefficients in arbitrary SAYD modules. Their "cup product of the second kind" agrees with Gorokhovsky's extended characteristic map, and therefore with the ConnesMoscovici characteristic map, when the coefficient module is $k_{(\sigma, \delta)}$. There are yet other ways of defining cup products in the context of Hopf equivariant Cuntz-Quillen formalism due to Crainic [5] and Nikonov-Sharygin [20], where the former constructs the cup product only for $C=H$ and for the 1-dimensional coefficient module $k_{(\sigma, \delta)}$, while the latter generalizes the construction to arbitrary $H$-module coalgebras and arbitrary SAYD coefficient modules. Our approach to products is different than all of the approaches we enumerated above in that we use the derived category of cyclic modules, while all the cup products we mentioned above use the theory of abstract cycles and closed graded traces (see $[\mathbf{2}$, p. 183], [17], [18, p. 74]) or the homotopy category of (special) towers of super complexes $[\mathbf{6}]$.

The last section of our paper is devoted to ramifications of an interesting technical problem in cyclic cohomology. There are essentially two different homotopical frameworks for the category cyclic modules:

(i) Connes' derived category of cyclic modules $[\mathbf{1}]$, and

(ii) Cuntz-Quillen formalism of homotopy category of towers of super complexes [6], which is equivalent to the derived category of mixed complexes $[\mathbf{1 3}]$ and the derived category of $S$-modules [14], thanks to Quillen [22].

One can see the difference in the simple fact that for a cyclic module $X_{\bullet}$, the derived functors $\operatorname{Ext}_{\Lambda}^{*}\left(k_{\bullet}^{\vee}, X_{\bullet}\right)$ in the category of cyclic modules compute the dual cyclic homology of $X$ • [16, Corollary 2.10 and Proposition 3.4], while the derived functors $\mathbf{E x t}_{\mathcal{M}}^{*}\left(\mathcal{B}_{*}\left(k_{\bullet}^{\vee}\right), \mathcal{B}_{*}\left(X_{\bullet}\right)\right)$ in the category of mixed complexes compute the negative cyclic homology of $X_{\bullet}$ [12, Theorem 2.3]. Here, $k_{\bullet}$ is the cocyclic module of the ground field viewed as a coalgebra, the dual cyclic homology of $X_{\bullet}$ is the cyclic cohomology of $X_{\bullet}^{\vee}$, the cyclic dual of a (co)cyclic module $X_{\bullet}$ defined by using Connes' duality functor [1] , and $\mathcal{B}_{*}\left(Z_{\bullet}\right)$ is the mixed complex of a (co)cyclic module $Z_{\bullet}$. The main result of this last section is Theorem 5.4, where we prove that replacing the derived category cyclic modules by the derived category of mixed complexes, or $S$-modules, or the homotopy category of towers of super complexes in Theorems 2.8, 2.11, and 3.2 will not change the pairings we have already defined. 
In this article $k$ will denote an arbitrary field. We make no assumption about the characteristic of $k$. We will use $H$ to denote a bialgebra, or a Hopf algebra with an invertible antipode over $k$, whenever necessary. All tensor products, unless otherwise explicitly stated, are over $k$.

\section{Equivariant actions of coalgebras on algebras}

In this section, $A$ will denote a unital associative left $H$-module algebra and $C$ will denote a counital coassociative left $H$-module coalgebra. Explicitly, one has

$$
h\left(a_{1} a_{2}\right)=\left(h_{(1)} a_{1}\right)\left(h_{(2)} a_{2}\right) \quad \text { and } \quad(h c)_{(1)} \otimes(h c)_{(2)}=h_{(1)} c_{(1)} \otimes h_{(2)} c_{(2)}
$$

for any $a_{1}, a_{2} \in A, c \in C$ and $h \in H$. We also assume

$$
h\left(1_{A}\right)=\varepsilon(h) 1_{A} \quad \text { and } \quad \varepsilon(h c)=\varepsilon(h) \varepsilon(c)
$$

for any $c \in C$ and $h \in H$. We will use $M$ to denote an arbitrary $H$-module/comodule with no assumption on the interaction between the $H$-module and $H$-comodule structures on $M$.

Given two morphisms $f_{1}, f_{2} \in \operatorname{Hom}_{k}(C, A)$, we define their convolution product as

$$
\left(f_{1} * f_{2}\right)(c):=f_{1}\left(c_{(1)}\right) f_{2}\left(c_{(2)}\right)
$$

for any $c \in C$. This binary operation on $\operatorname{Hom}_{k}(C, A)$ is an associative product. The unit for this algebra is $\eta(c):=\varepsilon(c) 1_{A}$. The proof of the following lemma is routine.

Lemma 2.1. There exists a morphism of algebras of the form $\alpha: A \rightarrow \operatorname{Hom}_{k}(C, A)$ if and only if one has a pairing $\phi: C \otimes A \rightarrow A$, which satisfies

$$
\phi\left(c, a_{1} a_{2}\right)=\phi\left(c_{(1)}, a_{1}\right) \phi\left(c_{(2)}, a_{2}\right) \quad \text { and } \quad \phi(c, 1)=\varepsilon(c) 1_{A}
$$

for any $c \in C$ and $a_{1}, a_{2} \in A$.

Definition 2.2. If one has a pairing between a module coalgebra $C$ and a module algebra $A$ as described in Lemma 2.1, then $C$ is said to act on $A$. Such an action is going to be called equivariant if one also has

$$
h \phi(c, a)=\phi(h c, a)
$$

for any $a \in A, h \in H$ and $c \in C$.

One can observe that an $H$-module coalgebra $C$ acts on an $H$-module algebra $A$ equivariantly, if and only if the canonical morphism of algebras $\alpha: A \rightarrow \operatorname{Hom}_{k}(C, A)$ factors through the inclusion $\operatorname{Hom}_{H}(C, A) \subseteq \operatorname{Hom}_{k}(C, A)[\mathbf{1 7}]$.

Definition 2.3. If $X_{\bullet}$ is a (para-)cocyclic $k$-module and $Y_{\bullet}$ is a (para-)cyclic $k$-module, then the graded module

$$
\operatorname{diag} \operatorname{Hom}_{k}\left(X_{\bullet}, Y_{\bullet}\right):=\bigoplus_{n} \operatorname{Hom}_{k}\left(X_{n}, Y_{n}\right)
$$


carries a (para-)cyclic module structure defined as

$$
\begin{aligned}
\left(\partial_{j} f\right)\left(x_{n-1}\right):=\partial_{j}^{Y} f\left(\partial_{j}^{X}\left(x_{n-1}\right)\right) & 0 \leqslant j \leqslant n, \\
\left(\sigma_{j} f_{n}\right)\left(x_{n+1}\right) & :=\sigma_{j}^{Y} f\left(\sigma_{j}^{X}\left(x_{n+1}\right)\right) \quad 0 \leqslant j \leqslant n, \\
\left(\tau_{n} f_{n}\right)\left(x_{n}\right) & :=\tau_{n, Y} f\left(\tau_{n, X}\left(x_{n}\right)\right)
\end{aligned}
$$

for any $f \in \operatorname{Hom}_{k}\left(X_{n}, Y_{n}\right), x_{i} \in X_{i}$ for $i=n-1, n, n+1$.

We will use $C y c_{\bullet}(X)$ to denote the classical cyclic $k$-module of an (co)associative (co)unital $k$-(co)algebra $X$. Also, we will use $k_{\bullet}$ to denote $C y c_{\bullet}\left(k^{c}\right)$, the cocyclic $k$-module of the ground field viewed as a coalgebra, and $k_{\bullet}^{\vee}$ to denote $C y c_{\bullet}(k)$, the cyclic $k$-module of the ground field $k$ viewed as an algebra. Note that in this case $k_{\bullet}^{\vee}$ is actually the cyclic dual of the cocyclic $k$-module $k_{\bullet}$ in the sense of [1, Lemme 1]. In general, if $X_{\bullet}$ is an arbitrary (co)cyclic $k$-module, then $X_{\bullet}^{\vee}$ will denote its cyclic dual. $T_{\bullet}(C, M)$ and $T_{\bullet}(A, M)$ are going to denote the para-(co)cyclic complex (also referred as "the cover complex") of the $H$-module coalgebra $C$ and $H$-module algebra $A$ with coefficients in a $H$-module/comodule $M$ respectively [16].

Here we recall the para-(co)cyclic structure morphisms on both $T_{\bullet}(C, M)$ and $T \bullet(A, M)$ from $[\mathbf{1 6}]$. The modules are defined as

$$
T_{n}(C, M):=C^{\otimes n+1} \otimes M \quad \text { and } \quad T_{n}(A, M):=A^{\otimes n+1} \otimes M .
$$

We define the structure morphisms on $T_{\bullet}(C, M)$ by

$$
\begin{aligned}
& \partial_{0}\left(c^{0} \otimes \cdots \otimes c^{n} \otimes m\right):=c_{(1)}^{0} \otimes c_{(2)}^{0} \otimes c^{1} \otimes \cdots \otimes c^{n} \otimes m, \\
& \sigma_{0}\left(c^{0} \otimes \cdots \otimes c^{n} \otimes m\right):=c^{0} \otimes \varepsilon\left(c^{1}\right) \otimes c^{2} \cdots \otimes c^{n} \otimes m, \\
& \tau_{n}\left(c^{0} \otimes \cdots \otimes c^{n} \otimes m\right):=c^{1} \otimes \cdots \otimes c^{n} \otimes m_{(-1)} c^{0} \otimes m_{(0)} .
\end{aligned}
$$

Then we define $\partial_{j}:=\tau_{n+1}^{-j} \partial_{0} \tau_{n}^{j}$ and $\sigma_{i}:=\tau_{n-1}^{-j} \sigma_{0} \tau_{n}^{j}$. Similarly, we let

$$
\begin{aligned}
& \partial_{0}\left(a_{0} \otimes \cdots \otimes a_{n} \otimes m\right):=a_{0} a_{1} \otimes a_{2} \otimes a_{n} \otimes m, \\
& \sigma_{0}\left(a_{0} \otimes \cdots \otimes a_{n} \otimes m\right):=a_{0} \otimes 1_{A} \otimes a_{1} \otimes \cdots \otimes a_{n} \otimes m, \\
& \tau_{n}\left(a_{0} \otimes \cdots \otimes a_{n} \otimes m\right):=S^{-1}\left(m_{(-1)}\right) a_{n} \otimes a_{0} \otimes \cdots \otimes a_{n-1} \otimes m_{(0)} .
\end{aligned}
$$

Then we define $\partial_{j}:=\tau_{n-1}^{j} \partial_{0} \tau_{n}^{-j}$ and $\sigma_{j}:=\tau_{n+1}^{j} \sigma_{0} \tau_{n}^{-j}$.

Proposition 2.4. Assume $C$ acts on $A$ equivariantly and let $M$ be an arbitrary $H$-module/comodule. Let us define

$$
\alpha_{n}\left(a_{0} \otimes \cdots \otimes a_{n}\right)\left(c^{0} \otimes \cdots \otimes c^{n} \otimes m\right):=\phi\left(c^{0}, a_{0}\right) \otimes \cdots \otimes \phi\left(c^{n}, a_{n}\right) \otimes m
$$

for any $n \geqslant 0, m \in M, a_{i} \in A$, and $c^{i} \in C$ for $i=0, \ldots, n$. Then $\alpha_{\bullet}$ defines a morphism of para-cyclic $k$-modules of the form

$$
\alpha_{\bullet}: C y c_{\bullet}(A) \rightarrow \operatorname{diag} \operatorname{Hom}_{H}\left(T_{\bullet}(C, M), T_{\bullet}(A, M)\right) .
$$


Proof. It is easy to observe that $\alpha_{n}$ is $H$-linear since the action of $C$ on $A$ is equivariant. Let us check if $\alpha$. now defines a morphism of para-cyclic modules:

$$
\begin{gathered}
\alpha_{n-1}\left(\partial_{0}^{A}\left(a_{0} \otimes \cdots \otimes a_{n}\right)\right)\left(c^{0} \otimes \cdots \otimes c^{n-1} \otimes m\right) \\
=\phi\left(c^{0}, a_{0} a_{1}\right) \otimes \phi\left(c^{1}, a_{2}\right) \otimes \cdots \otimes \phi\left(c^{n-1}, a_{n}\right) \otimes m \\
=\phi\left(c_{(1)}^{0}, a_{0}\right) \phi\left(c_{(2)}^{0}, a_{1}\right) \otimes \phi\left(c^{1}, a_{2}\right) \otimes \cdots \otimes \phi\left(c^{n-1}, a_{n}\right) \otimes m \\
=\partial_{0}^{(A, M)} \alpha_{n}\left(a_{0} \otimes \cdots \otimes a_{n}\right) \partial_{0}^{(C, M)}\left(c_{0} \otimes \cdots \otimes c^{n-1} \otimes m\right), \\
\alpha_{n+1}\left(\sigma_{0}^{A}\left(a_{0} \otimes \cdots \otimes a_{n}\right)\right)\left(c^{0} \otimes \cdots \otimes c^{n+1} \otimes m\right) \\
=\phi\left(c^{0}, a_{0}\right) \otimes \varepsilon\left(c_{1}\right) 1_{A} \otimes \phi\left(c^{2}, a_{1}\right) \otimes \cdots \otimes \phi\left(c^{n+1}, a_{n}\right) \otimes m \\
=\sigma_{0}^{(A, M)} \alpha_{n+1}\left(a_{0} \otimes \cdots \otimes a_{n}\right) \sigma_{0}^{(C, M)}\left(c^{0} \otimes \cdots \otimes c^{n+1} \otimes m\right), \\
\alpha_{n}\left(\tau_{n}\left(a_{0} \otimes \cdots \otimes a_{n}\right)\right)\left(c_{0} \otimes \cdots \otimes c_{n} \otimes m\right) \\
=\phi\left(c^{0}, a_{n}\right) \otimes \phi\left(c^{1}, a_{0}\right) \otimes \cdots \otimes \phi\left(c^{n}, a_{n-1}\right) \otimes m \\
\left.=S^{-1}\left(m_{(-1)}\right) \phi\left(m_{(-2)} c^{0}, a_{n}\right) \otimes \phi\left(c^{1}, a_{0}\right) \otimes \cdots \otimes \phi\left(c^{n}, a_{n-1}\right) \otimes m_{(0)}\right) \\
=\tau_{n,(A, M)}\left(\phi\left(c^{1}, a_{0}\right) \otimes \cdots \otimes \phi\left(c^{n}, a_{n-1}\right) \otimes \phi\left(m_{(-1)} c^{0}, a_{n}\right) \otimes m_{(0)}\right) \\
=\tau_{n,(A, M)} \alpha_{n}\left(a_{0} \otimes \cdots \otimes a_{n}\right) \tau_{(C, M)}\left(c_{0} \otimes \cdots \otimes c_{n} \otimes m\right),
\end{gathered}
$$

for any $c^{i} \in C, a_{i} \in A, m \in M$.

Remark 2.5. Now, we will recall a few relevant definitions from [16].

Let $J_{\bullet}(C, M)$ be the smallest para-cocyclic $k$-submodule and graded $H$-submodule (but not necessarily the para-cocyclic $H$-subcomodule) of $T_{\bullet}(C, M)$ generated by elements of the form $\left[L_{h}, \tau_{n}^{i}\right](\Psi)+\left(\tau_{n}^{n+1}-i d_{n}\right)(\Phi)$, where $\Psi, \Phi \in T_{n}(C, M), i \in \mathbb{Z}$, and $L_{h}$ is the graded $k$-module endomorphism of $T_{\bullet}(C, M)$ coming from the left diagonal action of $h \in H$ on $T_{n}(C, M)$ for each $n \geqslant 0$. One can similarly define $J_{\bullet}(A, M)$.

We define $Q_{\bullet}(C, M):=T_{\bullet}(C, M) / J_{\bullet}(C, M)$. One can see that $Q_{\bullet}(C, M)$ is a cocyclic $H$-module. Similarly, $Q_{\bullet}(A, M):=T_{\bullet}(A, M) / J_{\bullet}(A, M)$ is a cyclic $H$-module. This cocyclic (resp. cyclic) $H$-module is called the $H$-equivariant cocyclic (resp. cyclic) module of the pair $(C, M)$ (resp. $(A, M)$ ). The cyclic cohomology of the (co)cyclic $H$-modules $Q \bullet(C, M)$ and $Q \bullet(A, M)$ will be denoted by $H C_{H}^{*}(C, M)$ and $H C_{H}^{*}(A, M)$ respectively.

We define $C_{\bullet}(C, M):=k \otimes_{H} Q_{\bullet}(C, M)$. One can see that $C_{\bullet}(C, M)$ is a cocyclic $k$-module. Similarly, $C_{\bullet}(A, M):=k \otimes_{H} Q \bullet(A, M)$ is a cyclic $k$-module. This cocyclic (resp. cyclic) $k$-module is called the Hopf-cocyclic (resp. Hopf-cyclic) module of the pair $(C, M)$ (resp. $(A, M))$. The cyclic cohomology of the (co)cyclic $k$-modules $C_{\bullet}(C, M)$ and $C_{\bullet}(A, M)$ will be denoted by $H C_{\mathrm{Hopf}}^{*}(C, M)$ and $H C_{\mathrm{Hopf}}^{*}(A, M)$ respectively.

Lemma 2.6. For any $n \geqslant 0$ and $a_{i} \in A$ for $0 \leqslant i \leqslant n$, the restriction of the $H$-linear morphism $\alpha_{n}\left(a_{0} \otimes \cdots \otimes a_{n}\right)$ in $\operatorname{Hom}_{k}\left(T_{n}(C, M), T_{n}(A, M)\right)$ to $J_{n}(C, M)$ is a morphism in $\operatorname{Hom}_{H}\left(J_{n}(C, M), J_{n}(A, M)\right)$. 
Proof. It is sufficient to prove that for every $n \geqslant 0, a_{i} \in A$ with $0 \leqslant i \leqslant n$, the morphism $\alpha_{n}\left(a_{0} \otimes \cdots \otimes a_{n}\right)$ maps the elements of the form $\left[L_{h}, \tau_{n}^{i}\right](\Psi)+\left(\tau_{n}^{n+1}-i d_{n}\right)(\Phi)$ to elements of the same form. We know that each $\alpha_{n}\left(a_{0} \otimes \cdots \otimes a_{n}\right)$ is $H$-linear. So, we can reduce the proof to verification of the following string of equalities:

$$
\begin{aligned}
\alpha_{n}\left(a_{0} \otimes \cdots \otimes a_{n}\right) & \tau_{n,(C, M)}\left(c_{0} \otimes \cdots \otimes c_{n} \otimes m\right) \\
& =\alpha_{n}\left(a_{0} \otimes \cdots \otimes a_{n}\right)\left(c^{1} \otimes \cdots \otimes c^{n} \otimes m_{(-1)} c^{0} \otimes m_{(0)}\right) \\
& =\phi\left(c^{1}, a_{0}\right) \otimes \cdots \otimes \phi\left(c^{n}, a_{n-1}\right) \otimes m_{(-1)} \phi\left(c^{0}, a_{n}\right) \otimes m_{(0)} \\
& =\tau_{n,(A, M)}^{-1}\left(\phi\left(c^{0}, a_{n}\right) \otimes \phi\left(c^{1}, a_{0}\right) \otimes \cdots \otimes \phi\left(c^{n}, a_{n-1}\right) \otimes m\right)
\end{aligned}
$$

for $m \in M, a_{i} \in A, c^{i} \in C$ with $0 \leqslant i \leqslant n$.

Proposition 2.7. $\alpha_{\bullet}$ can be extended to a morphism of cyclic $k$-modules of the form

$$
\alpha_{\bullet}: C y c_{\bullet}(A) \rightarrow \operatorname{diag} \operatorname{Hom}_{H}(Q \bullet(C, M), Q \bullet(A, M))
$$

and

$$
\alpha_{\bullet}: C y c_{\bullet}(A) \rightarrow \operatorname{diag} \operatorname{Hom}_{k}(C \bullet(C, M), C \bullet(A, M)) .
$$

Proof. The first part of the statement follows from Lemma 2.6. The second part follows from the fact that $k \otimes_{H}(\cdot)$ is a functor from the category of left $H$-modules to the category of $k$-modules.

Theorem 2.8. The equivariant action of an $H$-module coalgebra $C$ on an $H$-module algebra $A$ induces a pairing of the form

$$
\smile: H C_{\mathrm{Hopf}}^{p}(C, M) \otimes H C_{\mathrm{Hopf}}^{q}(A, M) \rightarrow H C^{p+q}(A)
$$

for any $p, q \geqslant 0$.

Proof. First, we observe that we have

$$
H C_{\mathrm{Hopf}}^{p}(C, M)=\operatorname{Ext}_{\Lambda}^{p}\left(k_{\bullet}, C \bullet(C, M)\right)
$$

and

$$
H C_{\mathrm{Hopf}}^{q}(A, M)=\operatorname{Ext}_{\Lambda}^{q}\left(C \bullet(A, M), k_{\bullet}^{\vee}\right)
$$

We will use the Yoneda interpretation of Ext-groups [23], which was developed in [19, Chapter III]. Our approach in part is inspired by the use of Yoneda Ext-groups in [21]. In the Yoneda approach, one can represent the cohomology classes $\xi \in H C_{\mathrm{Hopf}}^{p}\left(k_{\bullet}, C_{\bullet}(C, M)\right)$ and $\nu \in H C_{\mathrm{Hopf}}^{q}\left(C \bullet(A, M), k_{\bullet}^{\vee}\right)$ by exact sequences of (co)cyclic $k$-modules

$$
\begin{array}{ll}
\xi: & 0 \leftarrow k_{\bullet} \leftarrow Y_{\bullet}^{1} \leftarrow \cdots \leftarrow Y_{\bullet}^{p} \leftarrow C_{\bullet}(C, M) \leftarrow 0, \\
\nu: & 0 \leftarrow C_{\bullet}(A, M) \leftarrow Z_{\bullet}^{1} \leftarrow \cdots \leftarrow Z_{\bullet}^{q} \leftarrow k_{\bullet}^{\vee} \leftarrow 0 .
\end{array}
$$

Since $k$ is a field, the functor $\operatorname{diag} \operatorname{Hom}_{k}(\cdot, C \bullet(A, M))$ from the category of cocyclic $k$-modules to the category of cyclic $k$-modules is exact. Hence we get an exact sequence $\operatorname{diag} \operatorname{Hom}_{k}(\xi, C \cdot(A, M))$ of the form

$$
\begin{aligned}
0 \leftarrow \operatorname{diag} \operatorname{Hom}_{k}\left(C_{\bullet}(C, M), C_{\bullet}(A, M)\right) \leftarrow \operatorname{diag} \operatorname{Hom}_{k}\left(Y_{\bullet}^{p}, C_{\bullet}(A, M)\right) \leftarrow \\
\cdots \leftarrow \operatorname{diag} \operatorname{Hom}_{k}\left(Y_{\bullet}^{1}, C_{\bullet}(A, M)\right) \leftarrow C_{\bullet}(A, M) \leftarrow 0,
\end{aligned}
$$

after observing the fact that $\operatorname{diag} \operatorname{Hom}_{k}\left(k_{\bullet}, C \bullet(A, M)\right)$ is isomorphic to $C_{\bullet}(A, M)$ as 
cyclic $k$-modules. Now, if we splice the exact sequences $\operatorname{diag} \operatorname{Hom}_{k}(\xi, C \bullet(A, M))$ and $\nu$, we get a class in $\operatorname{Ext}_{\Lambda}^{p+q}\left(\operatorname{diag}_{\operatorname{Hom}_{k}}\left(C_{\bullet}(C, M), C_{\bullet}(A, M)\right), k_{\bullet}^{\vee}\right)$. However, we also have a morphism of cyclic $k$-modules $\alpha$. constructed in Proposition 2.7. The result follows from the corresponding morphism of Ext-modules

$$
\begin{aligned}
\operatorname{Ext}_{\Lambda}^{p+q}\left(\alpha_{\bullet}, k_{\bullet}^{\vee}\right): \operatorname{Ext}_{\Lambda}^{p+q}\left(\operatorname{diag} \operatorname{Hom}_{k}(C \bullet(C, M), C \bullet(A, M)), k_{\bullet}^{\vee}\right) & \\
& \rightarrow \operatorname{Ext}_{\Lambda}^{p+q}\left(C y c_{\bullet}(A), k_{\bullet}^{\vee}\right),
\end{aligned}
$$

and then observing that $H C^{*}(A)=\operatorname{Ext}_{\Lambda}^{*}\left(C y c_{\bullet}(A), k_{\bullet}^{\vee}\right)$.

If one wishes to write a formula for this pairing, then one can write

$$
\xi \smile \nu:=\operatorname{Ext}_{\Lambda}^{p+q}\left(\alpha_{\bullet}, k_{\bullet}^{\vee}\right)\left(\operatorname{diag} \operatorname{Hom}_{k}\left(\xi, C_{\bullet}(A, M)\right) \circ \nu\right)
$$

for any $\xi \in H C_{\mathrm{Hopf}}^{p}(C, M)$ and $\nu \in H C_{\mathrm{Hopf}}^{q}(A, M)$, where $\circ$ denotes the Yoneda product in bivariant cohomology written in the opposite order; i.e.,

$$
\circ: \operatorname{Ext}_{\Lambda}^{p}\left(X_{\bullet}, Y_{\bullet}\right) \otimes \operatorname{Ext}_{\Lambda}^{q}\left(Y_{\bullet}, Z_{\bullet}\right) \rightarrow \operatorname{Ext}_{\Lambda}^{p+q}\left(X_{\bullet}, Z_{\bullet}\right) .
$$

However, there is one other pairing in the same setting. One can define this second pairing by the formula

$$
\nu \smile \xi:=\operatorname{Ext}_{\Lambda}^{p+q}\left(\alpha_{\bullet}, k_{\bullet}^{\vee}\right)\left(\operatorname{diag} \operatorname{Hom}_{k}(C \bullet(C, M), \nu) \circ \operatorname{diag} \operatorname{Hom}_{k}\left(\xi, k_{\bullet}^{\vee}\right)\right) .
$$

Below, we give an alternative construction for these pairings we gave above and prove that they actually are the same up to a sign.

Proposition 2.9. Assume that an $H$-module coalgebra $C$ acts on an $H$-module algebra $A$ equivariantly. Then, for any $\xi \in H C_{\mathrm{Hopf}}^{p}(C, M)$ and $\nu \in H C_{\mathrm{Hopf}}^{q}(A, M)$, one has $\xi \smile \nu=(-1)^{p q} \nu \smile \xi$.

Proof. Since the bifunctor $\operatorname{diag} \operatorname{Hom}_{k}(\cdot, \cdot \cdot)$ is exact in both variables, one has well-defined morphisms of the form

$$
\operatorname{diag} \operatorname{Hom}_{k}\left(Z_{\bullet}, \cdot\right): \operatorname{Ext}_{\Lambda}^{p}\left(X_{\bullet}, Y_{\bullet}\right) \rightarrow \operatorname{Ext}_{\Lambda}^{p}\left(\operatorname{diag} \operatorname{Hom}_{k}\left(Z_{\bullet}, X_{\bullet}\right), \operatorname{diag} \operatorname{Hom}_{k}\left(Z_{\bullet}, Y_{\bullet}\right)\right)
$$

and

$$
\operatorname{diag} \operatorname{Hom}_{k}\left(\cdot, Z_{\bullet}\right): \operatorname{Ext}_{\Lambda}^{p}\left(X_{\bullet}, Y_{\bullet}\right) \rightarrow \operatorname{Ext}_{\Lambda}^{p}\left(\operatorname{diag} \operatorname{Hom}_{k}\left(Y_{\bullet}, Z_{\bullet}\right), \operatorname{diag} \operatorname{Hom}_{k}\left(X_{\bullet}, Z_{\bullet}\right)\right)
$$

for any cocyclic modules $X_{\bullet}, Y_{\bullet}$ and cyclic module $Z_{\bullet}$. Since

$$
\xi \in H C_{\mathrm{Hopf}}^{p}(C, M):=\operatorname{Ext}_{\Lambda}^{p}\left(k_{\bullet}, C_{\bullet}(C, M)\right)
$$

and

$$
\nu \in H C_{\mathrm{Hopf}}^{q}(A, M):=\operatorname{Ext}_{\Lambda}^{q}\left(C_{\bullet}(A, M), k_{\bullet}^{\vee}\right),
$$

one has well-defined elements

$$
\zeta_{1}:=\operatorname{diag} \operatorname{Hom}_{k}(\xi, C \bullet(A, M)) \circ \nu,
$$

which belongs to $\operatorname{Ext}_{\Lambda}^{p+q}\left(\operatorname{diag} \operatorname{Hom}(C \bullet(C, M), C \bullet(A, M)), k_{\bullet}^{\vee}\right)$ and

$$
\zeta_{2}:=\operatorname{diag} \operatorname{Hom}_{k}\left(C_{\bullet}(C, M), \nu\right) \circ \operatorname{diag} \operatorname{Hom}_{k}\left(\xi, k_{\bullet}^{\vee}\right),
$$

which belongs to $\operatorname{Ext}_{\Lambda}^{p+q}\left(\operatorname{diag} \operatorname{Hom}\left(C \bullet(C, M), C_{\bullet}(A, M)\right), k_{\bullet}^{\vee}\right)$. Here we use the opposite composition notation as before. We observe that $\nu=\operatorname{diag} \operatorname{Hom}_{k}\left(k_{\bullet}, \nu\right)$. Then the 
proof that one has, $\zeta_{1}=(-1)^{p q} \zeta_{2}$ in $\operatorname{Ext}_{\Lambda}^{p+q}\left(\operatorname{diag} \operatorname{Hom}_{k}(C \bullet(C, M), C \bullet(A, M)), k_{\bullet}^{\vee}\right)$, reduces to proving that the bifunctor $\operatorname{diag} \operatorname{Hom}_{k}(\cdot, \cdot)$ satisfies the following property:

$$
\operatorname{diag} \operatorname{Hom}_{k}\left(a_{\bullet}, Y_{\bullet}^{\prime}\right) \circ \operatorname{diag} \operatorname{Hom}_{k}\left(X_{\bullet}, b_{\bullet}\right)=\operatorname{diag} \operatorname{Hom}_{k}\left(X_{\bullet}^{\prime}, b_{\bullet}\right) \circ \operatorname{diag} \operatorname{Hom}_{k}\left(a_{\bullet}, Y_{\bullet}\right)
$$

for any morphism of cocyclic modules $a_{\bullet}: X_{\bullet}^{\prime} \rightarrow X_{\bullet}$ and any morphism of cyclic modules $b_{\bullet}: Y_{\bullet} \rightarrow Y_{\bullet}^{\prime}$, which is obvious. The result follows after observing

$$
\xi \smile \nu:=\operatorname{Ext}_{\Lambda}^{p+q}\left(\alpha_{\bullet}, k_{\bullet}^{\vee}\right)\left(\zeta_{1}\right)=\operatorname{Ext}_{\Lambda}^{p+q}\left(\alpha_{\bullet}, k_{\bullet}^{\vee}\right)\left((-1)^{p q} \zeta_{2}\right):=(-1)^{p q} \nu \smile \xi,
$$

where $\alpha_{\bullet}$ is the morphism of cyclic modules we constructed in Proposition 2.4.

Theorem 2.10. The Connes-Moscovici characteristic map

$$
H C_{\mathrm{Hopf}}^{p}\left(H, k_{(\sigma, \delta)}\right) \rightarrow H C^{p}(A)
$$

defined in [3, Section VIII, Proposition 1] agrees with the pairing we defined in Theorem 2.8 for $C=H, M=k_{(\sigma, \delta)}$ and $q=0$. Here $k_{(\sigma, \delta)}$ denotes the 1-dimensional anti-Yetter-Drinfeld module of the module pair in involution $(\sigma, \delta)$.

Proof. Since $k_{(\sigma, \delta)}$ is a stable anti-Yetter-Drinfeld module (an SAYD module in short), by [15], we know that $C_{\bullet}\left(H, k_{(\sigma, \delta)}\right)$ is isomorphic to $k \otimes_{H} T_{\bullet}\left(H, k_{(\sigma, \delta)}\right)$. Therefore, one can identify $C_{\bullet}\left(H, k_{(\sigma, \delta)}\right)$ as the graded $k$-submodule of $T_{\bullet}\left(H, k_{(\sigma, \delta)}\right)$, which consists of elements the form $\sum_{i}\left(1 \otimes h_{i}^{1} \otimes \cdots \otimes h_{i}^{n}\right)$. The Connes-Moscovici characteristic is defined with the help of an invariant trace $\tau$ on $A$, which satisfies the following condition:

$$
\tau\left(h(a) a^{\prime}\right)=\tau\left(a S\left(h_{(1)}\right)\left(a^{\prime}\right) \delta\left(h_{(2)}\right)\right) \quad \text { and } \quad \tau(h(a))=\varepsilon(h) \tau(a)
$$

for any $a, a^{\prime} \in A$ and $h \in H$. This is equivalent to $\tau \in \operatorname{Hom}_{k}\left(A \otimes k_{(\sigma, \delta)}, k\right)$ being a cyclic cochain in degree 0 for the cyclic module $C_{\bullet}\left(A, k_{(\sigma, \delta)}\right)$. The characteristic map is defined as

$$
\gamma_{\bullet}\left(1 \otimes h^{1} \otimes \cdots \otimes h^{n}\right)\left(a_{0} \otimes \cdots \otimes a^{n}\right)=\tau\left(a_{0} h^{1}\left(a_{1}\right) \cdots h^{n}\left(a_{n}\right)\right)
$$

for any $1 \otimes h^{1} \otimes \cdots \otimes h^{n} \in C_{n}\left(H, k_{(\sigma, \delta)}\right)$ and $a_{0} \otimes \cdots \otimes a_{n} \in C y c_{n}(A)$. Now observe that one can write $\gamma_{\bullet}$ as a composition $\gamma_{\bullet}=\tau \circ \alpha_{\bullet}$, where $\alpha_{\bullet}$ is defined in the proof of Proposition 2.4. This means $\gamma_{\bullet}$ is the morphism,

$$
\operatorname{Hom}_{k}\left(\alpha_{\bullet}, k\right): \operatorname{Hom}_{k}\left(\operatorname{diag} \operatorname{Hom}_{k}(C \bullet(H, M), C \bullet(A, M)), k\right) \rightarrow \operatorname{Hom}_{k}\left(C y c_{\bullet}(A), k\right),
$$

where $M=k_{(\sigma, \delta)}$. Then $\operatorname{Hom}_{k}\left(\alpha_{\bullet}, k\right)$ induces the morphism $\operatorname{Ext}_{\Lambda}^{*}\left(\alpha_{\bullet}, k_{\bullet}^{\vee}\right)$ on cohomology, which is used in the proof of Theorem 2.8 to define the pairing. The result follows.

One can extend the pairing we defined above to the equivariant cyclic cohomology groups as follows:

Theorem 2.11. The equivariant action of an $H$-module coalgebra $C$ on an $H$-module algebra $A$ induces a pairing of the form

$$
\smile: H C_{H}^{p}(C, M) \otimes H C_{H}^{q}(A, M) \rightarrow \bigoplus_{r=0}^{p+q} H C^{p+q-r}(A) \otimes \operatorname{Ext}_{H}^{r}(k, k)
$$

for any $p, q \geqslant 0$. 
Proof. First observe that the morphism of cyclic modules $\alpha_{\bullet}$, defined in Proposition 2.4, can also be considered as a morphism of cyclic $H$-modules of the form $\alpha_{\bullet}: C y c_{\bullet}(A) \rightarrow \operatorname{diag} \operatorname{Hom}_{k}\left(Q_{\bullet}(C, M), Q_{\bullet}(A, M)\right)$. Here $C y c_{\bullet}(A)$ is considered as a trivial $H$-module and $\operatorname{diag} \operatorname{Hom}_{k}\left(Q \bullet(C, M), Q_{\bullet}(A, M)\right)$ has the following $H$-module structure:

$$
(h f)(\Psi):=h_{(1)} \cdot f\left(S\left(h_{(2)}\right) \cdot \Psi\right)
$$

for any $f \in \operatorname{diag} \operatorname{Hom}_{k}(Q \bullet(C, M), Q \bullet(A, M)), h \in H$ and $\Psi \in Q \bullet(C, M)$. We consider two cohomology classes

$$
\begin{array}{ll}
\mu: & 0 \leftarrow k_{\bullet} \leftarrow U_{\bullet}^{1} \leftarrow \cdots \leftarrow U_{\bullet}^{p} \leftarrow Q_{\bullet}(C, M) \leftarrow 0, \\
\nu: & 0 \leftarrow Q_{\bullet}(A, M) \leftarrow V_{\bullet}^{1} \leftarrow \cdots \leftarrow V_{\bullet}^{q} \leftarrow k_{\bullet}^{\vee} \leftarrow 0,
\end{array}
$$

elements of the cyclic cohomology modules $H C_{H}^{p}(C, M):=\operatorname{Ext}_{H[\Lambda]}^{p}\left(k_{\bullet}, Q \bullet(C, M)\right)$ and $H C_{H}^{q}(A, M):=\operatorname{Ext}_{H[\Lambda]}^{p}\left(Q_{\bullet}(A, M), k_{\bullet}\right)$, respectively. Now we consider the exact sequence $\operatorname{diag} \operatorname{Hom}_{k}\left(\mu, Q_{\bullet}(A, M)\right)$ of cyclic $H$-modules

$$
\begin{gathered}
0 \leftarrow \operatorname{diag} \operatorname{Hom}_{k}\left(Q \bullet(C, M), Q_{\bullet}(A, M)\right) \leftarrow \operatorname{diag} \operatorname{Hom}_{k}\left(U_{\bullet}^{p}, Q \bullet(A, M)\right) \leftarrow \\
\cdots \leftarrow \operatorname{diag} \operatorname{Hom}_{k}\left(U_{\bullet}^{1}, Q_{\bullet}(A, M)\right) \leftarrow Q_{\bullet}(A, M) \leftarrow 0 .
\end{gathered}
$$

We define a class in $\operatorname{Ext}_{H[\Lambda]}^{p+q}\left(\operatorname{diag} \operatorname{Hom}_{k}\left(Q_{\bullet}(C, M), Q_{\bullet}(A, M)\right), k_{\bullet}^{\vee}\right)$ by splicing the exact sequences $\operatorname{diag} \operatorname{Hom}_{k}\left(\mu, Q_{\bullet}(A, M)\right)$ and $\nu$ at $Q_{\bullet}(A, M)$. Then we define

$$
\mu \smile \nu \in \operatorname{Ext}_{H[\Lambda]}^{p+q}\left(C y c_{\bullet}(A), k_{\bullet}^{\vee}\right)
$$

by using the morphism of cyclic $H$-modules $\alpha_{\bullet}$. However, the $H$-module structure on $C y c_{\bullet}(A)$ is trivial. Therefore, using the first spectral sequence constructed in [16, Proposition 3.5] we obtain

$$
\operatorname{Ext}_{H[\Lambda]}^{p+q}\left(C y c_{\bullet}(A), k_{\bullet}^{\vee}\right) \cong \bigoplus_{r=0}^{p+q} H C^{p+q-r}(A) \otimes \operatorname{Hom}_{k}\left(\operatorname{Tor}_{r}^{H}(k, k), k\right) .
$$

Since $k$ is a field we have $\operatorname{Ext}_{H}^{r}(U, k) \cong \operatorname{Hom}_{k}\left(\operatorname{Tor}_{r}^{H}(U, k), k\right)$ for any $r \geqslant 0$ and any left $H$-module $U$, in particular, for $U=k$.

\section{Product (co)algebras}

We will change the notation to distinguish Hopf-cyclic cohomology and equivariant cyclic cohomology of $H$ - and $H \otimes H$-module algebras. We will use $Q \bullet(H ; A, M)$ to denote the cyclic $H$-module associated with the $H$-module algebra $A$ with coefficients in $M$. Also, we will use $H C_{\text {Hopf }}^{*}(H ; A, M)$ to denote the Hopf-cyclic cohomology of an $H$-module algebra $A$ with coefficients in $M$.

Proposition 3.1. Let $A, A^{\prime}$ be two $H$-module algebras and let $M$ and $M^{\prime}$ be two $H$-module/comodules. Then there is an external product structure on the equivariant 
cyclic cohomology groups

$$
H C_{H}^{p}(A, M) \otimes H C_{H}^{q}\left(A^{\prime}, M^{\prime}\right) \rightarrow H C_{H \otimes H}^{p+q}\left(A \otimes A^{\prime}, M \otimes M^{\prime}\right)
$$

and the Hopf-cyclic cohomology groups

$$
H C_{\mathrm{Hopf}}^{p}(H ; A, M) \otimes H C_{\mathrm{Hopf}}^{q}\left(H ; A^{\prime}, M^{\prime}\right) \rightarrow H C_{\mathrm{Hopf}}^{p+q}\left(H \otimes H ; A \otimes A^{\prime}, M \otimes M^{\prime}\right)
$$

for any $p, q \geqslant 0$.

Proof. Any cohomology class $\nu \in H C_{H}^{p}(A, M)$ and $\nu^{\prime} \in H C_{H}^{q}\left(A^{\prime}, M^{\prime}\right)$ can be represented by two exact sequences of cyclic $H$-modules of the form

$$
\nu: 0 \leftarrow Q \cdot(A, M) \leftarrow X_{\bullet}^{1} \leftarrow \cdots \leftarrow X_{\bullet}^{p} \leftarrow k_{\bullet}^{\vee} \leftarrow 0
$$

and

$$
\nu^{\prime}: 0 \leftarrow Q_{\bullet}\left(A^{\prime}, M^{\prime}\right) \leftarrow Y_{\bullet}^{1} \leftarrow \cdots \leftarrow Y_{\bullet}^{q} \leftarrow k_{\bullet}^{\vee} \leftarrow 0 .
$$

Now we define the external product $\nu \times \nu^{\prime}$ by the composition

$$
\begin{array}{r}
0 \leftarrow \operatorname{diag}\left(Q_{\bullet}(H ; A, M) \otimes_{k} Q_{\bullet}\left(H ; A^{\prime}, M^{\prime}\right)\right) \leftarrow \operatorname{diag}\left(X_{\bullet}^{1} \otimes_{k} Q_{\bullet}\left(H ; A^{\prime}, M^{\prime}\right)\right) \leftarrow \cdots \\
\leftarrow \operatorname{diag}\left(X_{\bullet}^{p} \otimes_{k} Q_{\bullet}\left(H ; A^{\prime}, M^{\prime}\right)\right) \leftarrow Y_{\bullet}^{1} \leftarrow \cdots \leftarrow Y_{\bullet}^{q} \leftarrow k_{\bullet}^{\vee} \leftarrow 0,
\end{array}
$$

which is an exact sequence of cyclic $H \otimes H$-modules. Here diag denotes the diagonal cyclic structure. Therefore, $\operatorname{diag}\left(U_{\bullet} \otimes V_{\bullet}\right)$ is a cyclic $H \otimes H$-module whenever $U_{\bullet}$ and $V_{\bullet}$ are cyclic $H$-modules. Now observe that there is a natural epimorphism from $Q \bullet\left(H \otimes H ; A \otimes A^{\prime}, M \otimes M^{\prime}\right)$ onto $\operatorname{diag}\left(Q \bullet(H ; A, M) \otimes Q \bullet\left(H ; A^{\prime}, M^{\prime}\right)\right)$. This proves our first assertion. The proof of our second assertion is similar.

Given two left $H$-comodules $M$ and $M^{\prime}$, we define

$$
M \square^{H} M^{\prime}:=\left\{\sum_{i} m_{i} \otimes m_{i}^{\prime} \mid \sum_{i} m_{i,(-1)} \otimes m_{i,(0)} \otimes m_{i}^{\prime}=\sum_{i} m_{i,(-1)}^{\prime} \otimes m_{i} \otimes m_{i,(0)}^{\prime}\right\} .
$$

Theorem 3.2. Let $A, A^{\prime}$ be two $H$-module algebras and let $M$ and $M^{\prime}$ be two $H$ module/comodules. Assume $H$ is cocommutative Hopf algebra and $M \square^{H} M^{\prime}$ is an $H$-submodule of $M \otimes M^{\prime}$. One has pairings of the form

$$
H C_{H}^{p}(A, M) \otimes H C_{H}^{q}\left(A^{\prime}, M^{\prime}\right) \rightarrow H C_{H}^{p+q}\left(A \otimes A^{\prime}, M \square^{H} M^{\prime}\right)
$$

and

$$
H C_{\mathrm{Hopf}}^{p}(H ; A, M) \otimes H C_{\mathrm{Hopf}}^{q}\left(H ; A^{\prime}, M^{\prime}\right) \rightarrow H C_{\mathrm{Hopf}}^{p+q}\left(H ; A \otimes A^{\prime}, M \square^{H} M^{\prime}\right)
$$

for any $p, q \geqslant 0$.

Now assume $Z$ is a $H$-comodule coalgebra; i.e., $Z$ is an $H$-comodule and a coalgebra such that the following compatibility condition is satisfied

$$
z_{[-1]} \otimes z_{[0](1)} \otimes z_{[0](2)}=z_{(1)[-1]} z_{(2)[-1]} \otimes z_{(1)[0]} \otimes z_{(2)[0]},
$$

where the $H$-comodule structure $\lambda: Z \rightarrow H \otimes Z$ is denoted by $z_{[-1]} \otimes z_{[0]}$, and the comultiplication $\Delta: Z \rightarrow Z \otimes Z$ is denoted by $z_{(1)} \otimes z_{(2)}$ for any $z \in Z$.

Lemma 3.3. Assume $H$ is commutative. If $Z$ and $Z^{\prime}$ are two arbitrary $H$-comodule coalgebras, then their product $Z \otimes Z^{\prime}$ has an $H$-comodule coalgebra structure. 
Definition 3.4. For a $H$-comodule coalgebra $Z$ and a stable $H$-module/comodule $M$, we define the Hopf-cyclic module $C_{\bullet}(Z, M)$ associated with the pair $(Z, M)$ as follows: on the graded $k$-module level we let $C_{n}(Z, M):=\operatorname{Hom}_{H \text {-comod }}\left(Z^{\otimes n+1}, M\right)$, where we view $Z^{\otimes n+1}$ as an $H$-comodule via the diagonal coaction; i.e.,

$$
\left(z^{0} \otimes \cdots \otimes z^{n}\right)_{[-1]} \otimes\left(z^{0} \otimes \cdots \otimes z^{n}\right)_{[0]}:=z_{[-1]}^{0} \cdots z_{[-1]}^{n} \otimes\left(z_{[0]}^{0} \otimes \cdots \otimes z_{[0]}^{n}\right)
$$

for any $z^{i} \in Z$. The cyclic structure morphisms are defined by

$$
\begin{aligned}
\left(\partial_{0} f\right)\left(z^{0} \otimes \cdots \otimes z^{n-1}\right) & :=f\left(z_{(1)}^{0} \otimes z_{(2)}^{0} \otimes z^{1} \otimes \cdots \otimes z^{n-1}\right), \\
\left(\sigma_{0} f\right)\left(z^{0} \otimes \cdots \otimes z^{n+1}\right) & :=\varepsilon\left(z^{1}\right) f\left(z^{0} \otimes z^{2} \otimes \cdots \otimes z^{n+1}\right), \\
\left(\tau_{n} f\right)\left(z^{0} \otimes \cdots \otimes z^{n}\right) & :=z_{[-1]}^{0} f\left(z^{1} \otimes \cdots \otimes z^{n} \otimes z_{[0]}^{0}\right)
\end{aligned}
$$

for any $f \in C_{n}(Z, M)$ and $z^{i} \in Z$. Then we set $\partial_{j}:=\tau_{n-1}^{j} \partial_{0} \tau_{n}^{-j}$ and $\sigma_{i}:=\tau_{n+1}^{i} \sigma_{0} \tau_{n}^{-i}$ for $0 \leqslant j \leqslant n$ and $0 \leqslant i \leqslant n$. The cyclic cohomology of this cyclic $H$-module will be denoted by $H C_{\text {Hopf }}^{*}(Z, M)$. The cyclic cohomology of the dual cocyclic object $C \bullet(Z, M)^{\vee}$ will be denoted by $H C_{\mathrm{Hopf}}^{\vee, *}(Z, M)$.

Theorem 3.5. Assume $Z$ and $Z^{\prime}$ are arbitrary $H$-comodule coalgebras and $M$ and $M^{\prime}$ are arbitrary $H$-module/comodules. If $H$ is commutative and $M$ is a symmetric $H$-module, then there is a pairing of the form

$$
H C_{\mathrm{Hopf}}^{\vee, p}(Z, M) \otimes H C_{\mathrm{Hopf}}^{\vee, q}\left(Z^{\prime}, M^{\prime}\right) \rightarrow H C_{\mathrm{Hopf}}^{\vee, p+q}\left(Z \otimes Z^{\prime}, M \otimes_{H} M^{\prime}\right)
$$

for any $p, q \geqslant 0$.

Proof. There is a well-defined morphism of cocyclic $k$-modules of the form

$$
*: \operatorname{diag}\left(C_{\bullet}(Z, M) \otimes C_{\bullet}\left(Z^{\prime}, M^{\prime}\right)\right) \rightarrow C_{\bullet}\left(Z \otimes Z^{\prime}, M \otimes_{H} M^{\prime}\right)
$$

given by the formula

$$
\left(f * f^{\prime}\right)\left(\left(x^{0}, y^{0}\right) \otimes \cdots \otimes\left(x^{n}, y^{n}\right)\right):=f\left(x^{0} \otimes \cdots \otimes x^{n}\right) \otimes_{H} f^{\prime}\left(y^{0} \otimes \cdots \otimes y^{n}\right)
$$

for any $n \geqslant 0, x^{i} \in Z$ and $y^{i} \in Z^{\prime}$. It is easy to see that $*$ is a morphism of simplicial modules. We see that

$$
\begin{aligned}
& \left(\tau_{n} f\right) *\left(\tau_{n} f^{\prime}\right)\left(\left(x^{0}, y^{0}\right) \otimes \cdots \otimes\left(x^{n}, y^{n}\right)\right) \\
& \quad=\left(\tau_{n} f\right)\left(x^{0} \otimes \cdots \otimes x^{n}\right) \otimes_{H}\left(\tau_{n} f^{\prime}\right)\left(y^{0} \otimes \cdots \otimes y^{n}\right) \\
& \quad=x_{[-1]}^{0} f\left(x^{1} \otimes \cdots \otimes x^{n} \otimes x_{[-1]}^{0}\right) \otimes_{H} y_{[-1]}^{0} f\left(y^{1} \otimes \cdots \otimes y^{n} \otimes y_{[-1]}^{0}\right) \\
& \quad=x_{[-1]}^{0} y_{[-1]}^{0} f\left(x^{1} \otimes \cdots \otimes x^{n} \otimes x_{[-1]}^{0}\right) \otimes_{H} f\left(y^{1} \otimes \cdots \otimes y^{n} \otimes y_{[-1]}^{0}\right) \\
& \quad=\left(\tau_{n}\left(f * f^{\prime}\right)\right)\left(\left(x^{0}, y^{0}\right) \otimes \cdots \otimes\left(x^{n}, y^{n}\right)\right)
\end{aligned}
$$

since $M$ is symmetric, as we wanted to show. Now take the exact sequences

$$
\begin{gathered}
\nu: 0 \leftarrow k_{\bullet}^{\vee} \leftarrow U_{\bullet}^{1} \leftarrow \cdots \leftarrow U_{\bullet}^{p} \leftarrow C_{\bullet}(Z, M) \leftarrow 0 \\
\nu^{\prime}: 0 \leftarrow k_{\bullet}^{\vee} \leftarrow V_{\bullet}^{1} \leftarrow \cdots \leftarrow V_{\bullet}^{q} \leftarrow C_{\bullet}\left(Z^{\prime}, M^{\prime}\right) \leftarrow 0
\end{gathered}
$$

representing two cyclic cohomology classes in $H C_{\mathrm{Hopf}}^{\vee, p}(Z, M)$ and $H C_{\mathrm{Hopf}}^{\vee, q}\left(Z^{\prime}, M^{\prime}\right)$ 
respectively. Consider the exact sequence

$$
\begin{aligned}
0 \leftarrow k_{\bullet}^{\vee} \leftarrow U_{\bullet}^{1} \leftarrow & \cdots \leftarrow U_{\bullet}^{p} \leftarrow \operatorname{diag}\left(C_{\bullet}(Z, M) \otimes V_{\bullet}^{1}\right) \leftarrow \cdots \\
& \leftarrow \operatorname{diag}\left(C_{\bullet}(Z, M) \otimes V_{\bullet}^{q}\right) \leftarrow \operatorname{diag}\left(C_{\bullet}(Z, M) \otimes C_{\bullet}\left(Z^{\prime}, M^{\prime}\right)\right) \leftarrow 0,
\end{aligned}
$$

which represents a class in $\operatorname{Ext}_{\Lambda}^{p+q}\left(k_{\bullet}^{\vee}, \operatorname{diag}\left(C_{\bullet}(Z, M) \otimes C_{\bullet}\left(Z^{\prime}, M^{\prime}\right)\right)\right)$. Using the morphism $*$ we defined above, we get a class in $\operatorname{Ext}_{\Lambda}^{p+q}\left(k_{\bullet}^{\vee}, C_{\bullet}\left(Z \otimes Z^{\prime}, M \otimes_{H} M^{\prime}\right)\right.$. The result follows after observing the fact that

$$
\operatorname{Ext}_{\Lambda}^{*}\left(k_{\bullet}^{\vee}, X_{\bullet}\right) \cong H C^{\vee, p}\left(X_{\bullet}\right)
$$

for any cyclic $k$-module $X_{\bullet}$.

\section{Crossed product (co)algebras}

In this section, we will assume $A$ is an $H$-module algebra and $B$ is an $H$-comodule algebra. $M$ will denote an arbitrary (left-left) stable anti-Yetter-Drinfeld (SAYD) module [10]; i.e., $M$ satisfies

$$
m_{(-1)} m_{(0)}=m \quad \text { and } \quad(h m)_{(-1)} \otimes(h m)_{(0)}=h_{(1)} m_{(-1)} S^{-1}\left(h_{(3)}\right) \otimes h_{(2)} m_{(0)}
$$

for any $m \in M$ and $h \in H$.

Definition 4.1. We construct a new algebra $A \rtimes B$, which is defined as $A \otimes B$ on the $k$-module level. The multiplication structure is defined by the formula

$$
(a, b)\left(a^{\prime}, b^{\prime}\right):=\left(a\left(b_{(-1)} a^{\prime}\right), b_{(0)} b^{\prime}\right)
$$

for $(a, b),\left(a^{\prime}, b^{\prime}\right) \in A \rtimes B$.

Recall the following definition from $[\mathbf{9}]$.

Definition 4.2. The Hopf-cocyclic $k$-module $C_{\bullet}(B, M)$ associated with the pair $(B, M)$ is defined by $C_{n}(B, M)=\operatorname{Hom}_{H \text {-comod }}\left(B^{\otimes n+1}, M\right)$ on the graded module level for any $n \geqslant 0$. This means that $f: B^{\otimes n+1} \rightarrow M$ is in $C_{n}(B, M)$ if and only if

$$
\left(f\left(b^{0} \otimes \cdots \otimes b^{n}\right)\right)_{(-1)} \otimes\left(f\left(b^{0} \otimes \cdots \otimes b^{n}\right)\right)_{(0)}=b_{(-1)}^{0} \cdots b_{(-1)}^{n} \otimes f\left(b_{(0)}^{0} \otimes \cdots \otimes b_{(0)}^{n}\right)
$$

for any $b^{i} \in B$ for $i=0, \ldots, n$. We let

$$
\begin{aligned}
\left(\partial_{0} f\right)\left(b^{0} \otimes \cdots \otimes b^{n+1}\right) & :=f\left(b^{0} b^{1} \otimes b^{2} \otimes \cdots \otimes b^{n+1}\right), \\
\left(\sigma_{0} f\right)\left(b^{0} \otimes \cdots \otimes b^{n-1}\right) & :=f\left(b^{0} \otimes 1_{B} \otimes b^{1} \otimes \cdots \otimes b^{n+1}\right), \\
\left(\tau_{n} f\right)\left(b^{0} \otimes \cdots \otimes b^{n}\right) & :=S\left(b_{(-1)}^{n}\right) f\left(b_{(0)}^{n} \otimes b^{1} \otimes \cdots \otimes b^{n-1}\right) .
\end{aligned}
$$

Then we define $\partial_{j}:=\tau_{n+1}^{-j} \partial_{0} \tau_{n}^{j}$ and $\sigma_{i}:=\tau_{n-1}^{-j} \sigma_{0} \tau_{n}^{j}$ for $0 \leqslant j \leqslant n+1$ and $0 \leqslant i \leqslant n$. Note that since $M$ is stable, $\tau_{n}^{n+1}=i d$ for any $n \geqslant 0$. 
Proposition 4.3. Let us define

$$
\begin{gathered}
\beta_{n}\left(\left(a_{0}, b^{0}\right) \otimes \cdots \otimes\left(a_{n}, b^{n}\right)\right)(f):=a_{0} \otimes b_{(-n)}^{0} a_{1} \otimes b_{(-n+1)}^{0} b_{(-n+1)}^{1} a_{2} \otimes \cdots \otimes b_{(-1)}^{0} \cdots b_{(-1)}^{n-1} a_{n} \\
\otimes f\left(b_{(0)}^{0} \otimes \cdots \otimes b_{(0)}^{n-1} \otimes b^{n}\right)
\end{gathered}
$$

for any $n \geqslant 0,\left(a_{i}, b^{i}\right) \in A \rtimes B$ and $f \in C_{n}(B, M)$. Then $\beta_{\bullet}$ defines a morphism of cyclic modules of the form

$$
\beta_{\bullet}: C y c_{\bullet}(A \rtimes B) \rightarrow \operatorname{diag} \operatorname{Hom}_{k}(C \bullet(B, M), C \bullet(A, M)) .
$$

Proof. We see that

$$
\begin{aligned}
& \beta_{n}\left(\partial_{0}\left(\left(a_{0}, b^{0}\right) \otimes \cdots \otimes\left(a_{n}, b^{n}\right)\right)\right)(f) \\
& =a_{0} b_{(-n)}^{0}\left(a_{1}\right) \otimes b_{(-n+1)}^{0} b_{(-n+1)}^{1} a_{2} \otimes \cdots \otimes b_{(-1)}^{0} \cdots b_{(-1)}^{n-1} a_{n} \\
& \quad \otimes f\left(b_{(0)}^{0} b_{(0)}^{1} \otimes b_{(0)}^{2} \otimes \cdots \otimes b_{(0)}^{n-1} \otimes b^{n}\right) \\
& =\partial_{0}\left(a_{0} \otimes b_{(-n)}^{0} a_{1} \otimes b_{(-n+1)}^{0} b_{(-n+1)}^{1} a_{2} \otimes \cdots \otimes b_{(-1)}^{0} \cdots b_{(-1)}^{n-1} a_{n}\right) \\
& \otimes\left(\partial_{0} f\right)\left(b_{(0)}^{0} \otimes \cdots \otimes b_{(0)}^{n-1} \otimes b^{n}\right), \\
& \beta_{n}\left(\sigma_{0}\left(\left(a_{0}, b^{0}\right) \otimes \cdots \otimes\left(a_{n}, b^{n}\right)\right)\right)(f) \\
& =a_{0} \otimes 1 \otimes b_{(-n)}^{0} a_{1} \otimes b_{(-n+1)}^{0} b_{(-n+1)}^{1} a_{2} \otimes \cdots \otimes b_{(-1)}^{0} \cdots b_{(-1)}^{n-1} a_{n} \\
& \otimes f\left(b_{(0)}^{0} \otimes 1 \otimes b_{(0)}^{1} \otimes \cdots \otimes b_{(0)}^{n-1} \otimes b^{n}\right) \\
& =\sigma_{0}\left(a_{0} \otimes b_{(-n)}^{0} a_{1} \otimes b_{(-n+1)}^{0} b_{(-n+1)}^{1} a_{2} \otimes \cdots \otimes b_{(-1)}^{0} \cdots b_{(-1)}^{n-1} a_{n}\right) \\
& \otimes\left(\sigma_{0} f\right)\left(b_{(0)}^{0} \otimes \cdots \otimes b_{(0)}^{n-1} \otimes b^{n}\right),
\end{aligned}
$$

and finally we obtain

$$
\begin{aligned}
& \beta_{n}\left(\tau_{n}\left(\left(a_{0}, b^{0}\right) \otimes \cdots \otimes\left(a_{n}, b^{n}\right)\right)\right)(f) \\
& =a_{n} \otimes b_{(-n)}^{n} a_{0} \otimes b_{(-n+1)}^{n} b_{(-n+1)}^{0} a_{1} \otimes \cdots \otimes b_{(-1)}^{n} b_{(-1)}^{0} \cdots b_{(-1)}^{n-2} a_{n-1} \\
& \otimes f\left(b_{(0)}^{n} \otimes b_{(0)}^{0} \otimes \cdots \otimes b_{(0)}^{n-2} \otimes b^{n-1}\right) \\
& =S^{-1}\left(b_{(-2)}^{n}\right) a_{n} \otimes a_{0} \otimes b_{(-n+1)}^{0} a_{1} \otimes \cdots \otimes b_{(-1)}^{0} \cdots b_{(-1)}^{n-2} a_{n-1} \\
& \otimes S\left(b_{(-1)}^{n}\right) f\left(b_{(0)}^{n} \otimes b_{(0)}^{0} \otimes \cdots \otimes b_{(0)}^{n-2} \otimes b^{n-1}\right),
\end{aligned}
$$

where the last equality follows from the fact that $C_{\bullet}(A, M)$ is a trivial $H$-module with respect to the diagonal $H$-action. Then, using the fact that $M$ is a SAYD module and $f \in \operatorname{Hom}_{H \text {-comod }}\left(B^{\otimes n+1}, M\right)$, we get

$$
\begin{aligned}
\beta_{n}\left(\tau_{n}(\right. & \left.\left.\left(a_{0}, b^{0}\right) \otimes \cdots \otimes\left(a_{n}, b^{n}\right)\right)\right)(f) \\
= & S^{-1}\left(b_{(-1)(1)}^{n}\right) S^{-1}\left(b_{(0)(-1)}^{n} b_{(0)(-1)}^{0} \cdots b_{(0)(-1)}^{n-2} b_{(-1)}^{n-1}\right) b_{(-1)(3)}^{n} b_{(-1)}^{0} \cdots b_{(-1)}^{n-1} a_{n} \\
& \otimes a_{0} \otimes b_{(-n)}^{0} a_{1} \otimes \cdots \otimes b_{(-2)}^{0} \cdots b_{(-2)}^{n-2} a_{n-1} \otimes S\left(b _ { ( - 1 ) ( 2 ) } ^ { n } f \left(b_{(0)(0)}^{n}\right.\right. \\
& \left.\otimes b_{(0)(0)}^{0} \otimes \cdots \otimes b_{(0)(0)}^{n-2} \otimes b_{(0)}^{n-1}\right) \\
= & \tau_{n}\left(\beta_{n}\left(\left(a_{0}, b_{0}\right) \otimes \cdots \otimes\left(a_{n}, b_{n}\right)\right)\left(\tau_{n} f\right)\right),
\end{aligned}
$$

as we wanted to show. 
Theorem 4.4. Assume $M$ is a SAYD module over $H$. If $A$ is an $H$-module algebra and $B$ is an $H$-comodule algebra, then there is a pairing of the form

$$
\smile: H C_{\mathrm{Hopf}}^{p}(A, M) \otimes H C_{\mathrm{Hopf}}^{q}(B, M) \rightarrow H C^{p+q}(A \rtimes B)
$$

for any $p, q \geqslant 0$.

Proof. Assume we have two exact sequences

$$
\begin{gathered}
\nu: 0 \leftarrow k_{\bullet} \leftarrow V_{\bullet}^{1} \leftarrow \cdots \leftarrow V_{\bullet}^{p} \leftarrow C_{\bullet}(B, M) \leftarrow 0 \\
\nu^{\prime}: 0 \leftarrow C_{\bullet}(A, M) \leftarrow W_{\bullet}^{1} \leftarrow \cdots \leftarrow W_{\bullet}^{q} \leftarrow k_{\bullet}^{\vee} \leftarrow 0
\end{gathered}
$$

representing two cyclic classes $\nu \in H C_{\mathrm{Hopf}}^{p}(A, M)$ and $\nu^{\prime} \in H C_{\mathrm{Hopf}}^{q}(B, M)$. We construct a new exact sequence $\operatorname{diag} \operatorname{Hom}_{k}\left(\nu, C_{\bullet}(A, M)\right)$

$$
\begin{aligned}
& 0 \leftarrow \operatorname{diag} \operatorname{Hom}_{k}(C \bullet(C, M), C \bullet(A, M)) \leftarrow \operatorname{diag} \operatorname{Hom}_{k}\left(V_{\bullet}^{p}, C_{\bullet}(A, M)\right) \leftarrow \\
& \cdots \operatorname{diag} \operatorname{Hom}_{k}\left(V_{\bullet}^{1}, C_{\bullet}(A, M)\right) \leftarrow C \bullet(A, M) \leftarrow 0
\end{aligned}
$$

and then splice it with the exact sequence $\nu^{\prime}$ at $C_{\bullet}(A, M)$ to get a class in the cohomology module $H C^{p+q}\left(\operatorname{diag} \operatorname{Hom}_{k}\left(C_{\bullet}(B, M), C_{\bullet}(A, M)\right)\right)$. Now we use the mor-

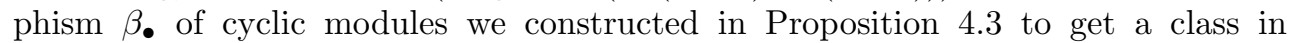
$H C^{p+q}(A \rtimes B)$.

Definition 4.5. Given an $H$-module coalgebra $C$ and an $H$-comodule coalgebra $Z$, we define the crossed product coalgebra $Z \ltimes C$ as follows: we let $Z \ltimes C:=Z \otimes C$ on the $k$-module level. The counit on $Z \ltimes C$ is the tensor product of counits on $C$ and $Z$ respectively. The comultiplication structure is defined by the formula

$$
(z, c)_{(1)} \otimes(z, c)_{(2)}:=\left(z_{(1)}, z_{(2)[-1]} c_{(1)}\right) \otimes\left(z_{(2)[0]}, c_{(2)}\right)
$$

for any $(c, z) \in Z \ltimes C$. For coassociativity, one must have

$$
\begin{aligned}
(z, c)_{(1)(1)} & \otimes(z, c)_{(1)(2)} \otimes(z, c)_{(2)} \\
& =\left(z_{(1)}, z_{(2)[-1]} c_{(1)}\right) \otimes\left(z_{(2)[0]}, c_{(2)}\right)_{(1)} \otimes\left(z_{(2)[0]}, c_{(2)}\right)_{(2)} \\
& =\left(z_{(1)}, z_{(2)[-1]} c_{(1)}\right) \otimes\left(z_{(2)[0](1)}, z_{(2)[0](2)[-1]} c_{(2)}\right) \otimes\left(z_{(2)[0](2)[0]}, c_{(3)}\right) .
\end{aligned}
$$

The compatibility conditions for comodule coalgebras give in equation (3.1) imply

$$
z_{[-1]} \otimes z_{[0](1)} \otimes z_{[0](2)[-1]} \otimes z_{[0](2)[0]}=z_{(1)[-1]} z_{(2)[-1]} \otimes z_{(1)[0]} \otimes z_{(2)[-1]} \otimes z_{(2)[0]} .
$$

This in turn yields

$$
\begin{aligned}
(z, c)_{(1)(1)} & \otimes(z, c)_{(1)(2)} \otimes(z, c)_{(2)} \\
& =\left(z_{(1)}, z_{(2)[-1]} z_{(3)[-1]} c_{(1)}\right) \otimes\left(z_{(2)[0]}, z_{(3)[-1]} c_{(2)}\right) \otimes\left(z_{(3)[0]}, c_{(3)}\right) \\
& =(z, c)_{(1)} \otimes(z, c)_{(2)(1)} \otimes(z, c)_{(2)(2)}
\end{aligned}
$$

for any $z \in Z$ and $c \in C$ as we wanted to show. 
Proposition 4.6. Assume $M$ is an SAYD module. Let us define $\xi_{n}$ by the formula

$$
\begin{aligned}
\xi_{n}\left(\left(z^{0}, c^{0}\right) \otimes \cdots \otimes\left(z^{n}, c^{n}\right)\right)(f) & \\
:= & S^{-1}\left(z_{[-1]}^{0} \cdots z_{[-1]}^{n}\right) c^{0} \otimes S^{-1}\left(z_{[-2]}^{1} \cdots z_{[-2]}^{n}\right) c^{1} \otimes \cdots \otimes S^{-1}\left(z_{[-n-1]}^{n}\right) c^{n} \\
& \otimes f\left(z_{[0]}^{0} \otimes \cdots \otimes z_{[0]}^{n}\right) \\
= & S^{-1}\left(z_{[-1]}^{0} \cdots z_{[-1]}^{n-1}\right) c^{0} \otimes S^{-1}\left(z_{[-2]}^{1} \cdots z_{[-2]}^{n-1}\right) c^{1} \otimes \cdots \otimes S^{-1}\left(z_{[-n]}^{n-1}\right) c^{n-1} \otimes c^{n} \\
& \otimes z_{[-1]}^{n} f\left(z_{[0]}^{0} \otimes \cdots \otimes z_{[0]}^{n}\right)
\end{aligned}
$$

for any $n \geqslant 0, c^{i} \in C, z^{i} \in C$ and $f \in C_{n}(Z, M)$. Then $\xi_{\bullet}$ is a morphism of cocyclic modules of the form

$$
\xi_{\bullet}: C y c_{\bullet}(Z \ltimes C) \rightarrow \operatorname{diag} \operatorname{Hom}_{k}\left(C \bullet(Z, M), C_{\bullet}(C, M)\right) .
$$

Proof. We will prove that $\xi_{\bullet}$ and the cyclic operators are compatible, but we will leave the verification of the fact that $\xi_{\bullet}$ is compatible with the face and degeneracy maps to the reader. We also observe

$$
\begin{aligned}
\xi_{n}( & \left(\tau_{n}\left(\left(z^{0}, c^{0}\right) \otimes \cdots \otimes\left(z^{n}, c^{n}\right)\right)\right)(f) \\
= & \xi_{n}\left(\left(z^{1}, c^{1}\right) \otimes \cdots \otimes\left(z^{n}, c^{n}\right) \otimes\left(z^{0}, c^{0}\right)\right)(f) \\
= & S^{-1}\left(z_{[-1]}^{1} \cdots z_{[-1]}^{n}\right) c^{1} \otimes S^{-1}\left(z_{[-2]}^{2} \cdots z_{[-2]}^{n}\right) c^{2} \otimes \cdots \otimes S^{-1}\left(z_{[-n]}^{n}\right) c^{n} \otimes c^{0} \\
& \otimes z_{[-1]}^{0} f\left(z_{[0]}^{1} \otimes \cdots \otimes z_{[0]}^{n} \otimes z_{[0]}^{0}\right) \\
= & S^{-1}\left(z_{[-2]}^{1} \cdots z_{[-2]}^{n}\right) c^{1} \otimes S^{-1}\left(z_{[-3]}^{2} \cdots z_{[-3]}^{n}\right) c^{2} \otimes \cdots \otimes S^{-1}\left(z_{[-n-1]}^{n}\right) c^{n} \\
& \otimes z_{[0][-4]}^{0} z_{[0][-1]}^{1} \cdots z_{[0][-1]}^{n} z_{[0][-1]}^{0} S^{-1}\left(z_{[0][-2]}^{0}\right) S^{-1}\left(z_{[-1]}^{0} z_{[-1]}^{1} \cdots z_{[-1]}^{n}\right) c^{0} \\
& \otimes z_{[0][-3]}^{0} f\left(z_{[0][0]}^{1} \otimes \cdots \otimes z_{[0][0]}^{n} \otimes z_{[0][0]}^{0}\right) \\
= & \tau_{n}\left(\xi_{n}\left(\left(z^{0}, c^{0}\right) \otimes \cdots \otimes\left(z^{n}, c^{n}\right)\right)\left(\tau_{n} f\right)\right)
\end{aligned}
$$

for any $n \geqslant 0, z^{i} \in Z, c^{i} \in C$ and $f \in C_{n}(Z, M)$.

Theorem 4.7. Fix an $S A Y D$ module $M$ over $H$. For an $H$-module coalgebra $C$ and an $H$-comodule coalgebra $Z$ one has a pairing of the form

$$
H C^{p}(Z \ltimes C) \otimes H C_{\mathrm{Hopf}}^{\vee, q}(Z, M) \rightarrow H C_{\mathrm{Hopf}}^{p+q}(C, M)
$$

for any $p, q \geqslant 0$, where $H C_{\mathrm{Hopf}}^{\vee, *}(Z, M)$ denotes the cyclic cohomology of the dual (cocyclic) module of the cyclic $k$-module $C \bullet(Z, M)$.

Proof. Any class $[\nu]$ in $H C_{\mathrm{Hopf}}^{\vee, q}(Z, M)$ is represented by an exact sequence of the form $\nu: 0 \leftarrow k_{\bullet}^{\vee} \leftarrow X_{\bullet}^{1} \leftarrow \cdots \leftarrow X_{\bullet}^{q} \leftarrow C_{\bullet}(Z, M) \leftarrow 0$. Now one can construct a new class $\operatorname{diag} \operatorname{Hom}_{k}\left(\nu, C_{\bullet}(C, M)\right)$ in $\operatorname{Ext}_{\Lambda}^{q}\left(\operatorname{diag} \operatorname{Hom}_{k}\left(C \bullet(Z, M), C_{\bullet}(C, M)\right), C_{\bullet}(C, M)\right)$ via

$$
\begin{aligned}
0 \leftarrow \operatorname{diag} \operatorname{Hom}_{k}\left(C_{\bullet}(Z, M), C_{\bullet}(C, M)\right) \leftarrow \operatorname{diag} \operatorname{Hom}_{k}\left(X_{\bullet}^{q}, C_{\bullet}(C, M)\right) \leftarrow \cdots \\
\leftarrow \operatorname{diag} \operatorname{Hom}_{k}\left(X_{\bullet}^{1}, C_{\bullet}(C, M)\right) \leftarrow C_{\bullet}(C, M) \leftarrow 0 .
\end{aligned}
$$

By using $\xi_{\bullet}$, which we defined in Proposition 4.6, we obtain a class in

$$
\operatorname{Ext}_{\Lambda}^{q}\left(C y c_{\bullet}(Z \ltimes C), C_{\bullet}(C, M)\right) .
$$

In other words, we have a morphism of graded modules of the form

$$
H C_{\mathrm{Hopf}}^{\vee, *}(Z, M) \rightarrow \operatorname{Ext}_{\Lambda}^{*}\left(C y c_{\bullet}(Z \ltimes C), C_{\bullet}(C, M)\right) .
$$


Now, pairing with the cyclic cohomology of the crossed product coalgebra

$$
H C^{p}(Z \ltimes C):=\operatorname{Ext}_{\Lambda}^{p}\left(k_{\bullet}, C y c_{\bullet}(Z \ltimes C)\right)
$$

and using the Yoneda composition, we get the desired cup product.

\section{Cup products in the derived category of mixed complexes}

Recall that in the construction of various products, we heavily relied on the exact bifunctor $\operatorname{diag} \operatorname{Hom}_{k}(\cdot, \cdot)$, which takes a pair of cocyclic and cyclic $k$-modules as an input and which produces a cyclic $k$-module. Also, we explicitly used the derived category of (co)cyclic $k$-modules or $H$-modules depending on whether we needed the bivariant Hopf or the equivariant bivariant cyclic cohomology. An alternative approach would be to develop a similar theory by using the derived category of mixed complexes, or towers of super complexes. But, thanks to Quillen [22] we know that the category of (special) towers of super complexes is homotopy equivalent to the category of mixed complexes and the category of $S$-modules. So, it is enough to develop similar cup products in the derived category of mixed complexes. To this end, we need to mimic the construction of the exact bifunctor $\operatorname{diag} \operatorname{Hom}_{k}(\cdot, \cdot)$ within the framework of mixed complexes.

One can view the category of mixed complexes as the category of differential graded $\mathcal{M}$-modules, where $\mathcal{M}$ is the free graded symmetric algebra of the graded vector space $k$, and where the single generator is assumed to have degree 1 . We will suggestively use $B \in \mathcal{M}$ to denote this generator. Then the derived category of differential graded $\mathcal{M}$-modules is the homotopy category of mixed complexes [12].

Lemma 5.1 ([21, Proposition 1.5]). The functor $\mathcal{B}_{*}:$ mod- $\Lambda \rightarrow \mathcal{M}$-dgmod, which sends a cyclic module to its mixed complex, is an exact functor. Therefore it induces natural morphisms of derived bifunctors

$$
\operatorname{Ext}_{\Lambda}^{*}(\cdot, \cdot) \rightarrow \boldsymbol{E x t}_{\mathcal{M}}^{*}(\cdot, \cdot),
$$

where $\boldsymbol{E x t}_{\mathcal{M}}$ stands for the derived functor of the Hom-bifunctor of the category of differential graded $\mathcal{M}$-modules.

A bi-differential graded $k$-module $\left(X_{*, *} ; b_{1}, b_{2}\right)$ is called a mixed double complex if there exists differentials $B_{1}$ and $B_{2}$ of degree 1 such that

$$
\left[b_{i}, b_{j}\right]=\left[b_{i}, B_{j}\right]=\left[B_{i}, B_{j}\right]=0 \quad \text { whenever } \quad i \neq j
$$

and

$$
b_{i} B_{i}+B_{i} b_{i}=0 \text { for } i=1,2 .
$$

For any mixed double complex $\left(X_{*, *} ; b_{1}, b_{2}, B_{1}, B_{2}\right)$ we will use $\operatorname{Tot}_{*}\left(X_{*, *}\right)$ to denote the mixed complex over the total complex of the bi-differential graded module $X_{*, *}$ with the degree 1 differential $B=B_{1}+B_{2}$.

Lemma 5.2. Assume $X_{\bullet}$ is an arbitrary cocyclic $k$-module and $Y_{\bullet}$ is an arbitrary cyclic $k$-module. Then in the derived category of mixed complexes there is an isomorphism of the form

$$
\eta_{*}: \operatorname{Tot}_{*} \operatorname{Hom}_{k}\left(\mathcal{B}_{*}\left(X_{\bullet}\right), \mathcal{B}_{*}\left(Y_{\bullet}\right)\right) \rightarrow \mathcal{B}_{*}\left(\operatorname{diag} \operatorname{Hom}_{k}\left(X_{\bullet}, Y_{\bullet}\right)\right) .
$$


Proof. We know that $\operatorname{Hom}_{k}\left(X_{\bullet}, Y_{\bullet}\right)$ is a bi-cyclic $k$-module. If this bi-cyclic module was a product of two cyclic modules, then we could have used [11]. Unless $X_{n}$ is finite dimensional for any $n \geqslant 0$, this is not the case. But, we can still use [7, Theorem 3.1] with $T=i d$ to get the desired isomorphism $\eta_{*}$.

Lemma 5.3. Let $M$ be an arbitrary $H$-module/comodule. Assume $A$ is an $H$-module algebra, $B$ is an $H$-comodule algebra and $C$ is a $H$-module coalgebra. Then there are morphisms of mixed complexes of the form

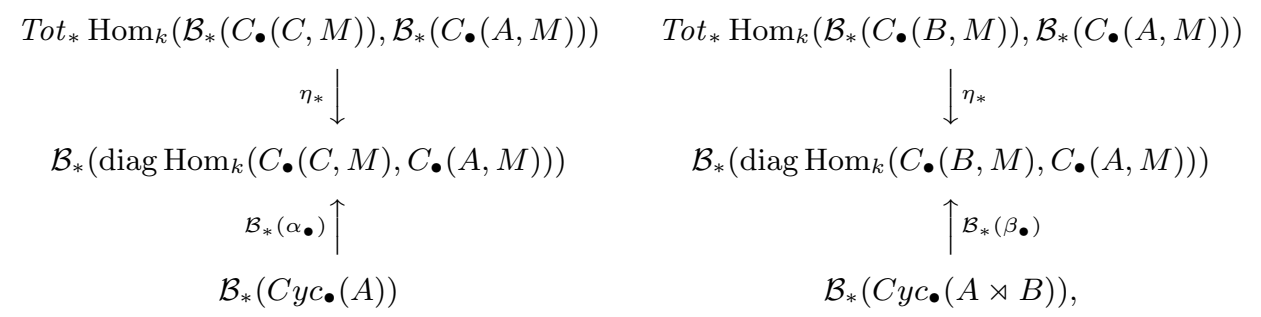

where on the second column, we also require $M$ to be SAYD.

Proof. The upward arrows come from applying the functor $\mathcal{B}_{*}$ to $\alpha_{\bullet}$ that we constructed in Propositions 2.4 and 2.7, and to $\beta$ • which was constructed in Proposition 4.3. The downward arrows come from Lemma 5.2.

Theorem 5.4. One can define pairings analogous to the pairings we defined in Theorems 2.8, 2.11 and 3.2 by using the derived category of mixed complexes instead of the derived category of cyclic modules. However, these pairings defined in the derived category of mixed complexes agree with those defined in the derived category of cyclic modules.

Proof. The fact that $\eta_{*}$ is an isomorphism in the derived category of mixed complexes allows us to use $\operatorname{Tot}_{*} \operatorname{Hom}_{k}\left(\mathcal{B}_{*}(\cdot), \mathcal{B}_{*}(\cdot)\right)$ as a replacement for $\operatorname{diag} \operatorname{Hom}_{k}(\cdot, \cdot)$ in the category of mixed complexes. Then Lemma 5.1 gives us a comparison map between these pairings, which are isomorphisms for the specific cases we enumerate.

Remark 5.5. One can prove a version of Lemma 5.2 for the case $X_{\bullet}$ is a cyclic and $Y_{\bullet}$ is a cocyclic module, provided one reverses the roles of the Hochschild differential $b$ and the differential $B$ for cocyclic modules. In other words, for a cocyclic module $Y_{\bullet}$, we consider $\left(\mathcal{B}_{*}\left(Y_{\bullet}\right), B\right)$ as the differential graded $\mathcal{M}$-module via the action of the Hochschild differential. Then one can define pairings in the derived category of mixed complexes analogous to the pairings we obtained in Theorems 3.5 and 4.7, if one replaces dual cyclic cohomology functors with negative cyclic cohomology and dual negative cyclic cohomology functors respectively.

\section{Acknowledgements}

This article was conceived during my stay in Warsaw in November and December of 2006 as a part of Transfer of Knowledge Programme in Noncommutative Geometry and Quantum Groups. I was partially supported by Marie Curie Fellowship MKTD-CT-2004-509794. I would like to thank the Department of Physics of Warsaw University and the Banach Center for their invitations, warm hospitality and support. 
I would especially like to thank Piotr M. Hajac for suggesting that I give a talk about cup products and characteristic map in Hopf-cyclic cohomology in his Noncommutative Geometry Seminar, and our ensuing discussions on Hopf-cyclic cohomology.

I would also like to thank the referee for his/her constructive review of the article, which improved the key aspects of our presentation.

\section{References}

[1] A. Connes, Cohomologie cyclique et foncteurs $\mathrm{Ext}^{n}$, C. R. Acad. Sci. Paris Sér. I Math. 296 (1983), no. 23, 953-958.

[2] A. Connes, Noncommutative geometry, Academic Press Inc., New York, 1994, http://www . alainconnes . org/docs/book94bigpdf .pdf.

[3] A. Connes and H. Moscovici, Hopf algebras, cyclic cohomology and transverse index theorem, Comm. Math. Phys. 198 (1998), no. 1, 199-246.

[4] A. Connes and H. Moscovici, Cyclic cohomology and Hopf algebras (in memory of Moshé Flato (1937-1998), Lett. Math. Phys. 48 (1999), no. 1, 97-108.

[5] M. Crainic, Cyclic cohomology of Hopf algebras, J. Pure Appl. Algebra 166 (2002), no. 1-2, 29-66.

[6] J. Cuntz and D. Quillen, Cyclic homology and nonsingularity, J. Amer. Math. Soc. 8 (1995), no. 2, 373-442.

[7] E. Getzler and J.D.S. Jones, The cyclic homology of crossed product algebras, J. Reine Angew. Math. 445 (1993), 161-174.

[8] A. Gorokhovsky, Secondary characteristic classes and cyclic cohomology of Hopf algebras, Topology 41 (2002), no. 5, 993-1016.

[9] P.M. Hajac, M. Khalkhali, B. Rangipour, and Y. Sommerhäuser, Hopf-cyclic homology and cohomology with coefficients, C. R. Math. Acad. Sci. Paris. 338 (2004), no. 9, 667-672.

[10] P.M. Hajac, M. Khalkhali, B. Rangipour, and Y. Sommerhäuser, Stable antiYetter-Drinfeld modules, C. R. Math. Acad. Sci. Paris 338 (2004), no. 8, $587-590$.

[11] C.E. Hood and J.D.S. Jones, Some algebraic properties of cyclic homology groups, K-Theory 1 (1987), no. 4, 361-384.

[12] J.D.S. Jones and C. Kassel, Bivariant cyclic theory, K-Theory 3 (1989), no. 4, 339-365.

[13] C. Kassel, Cyclic homology, comodules, and mixed complexes, J. Algebra 107 (1987), no. 1, 195-216.

[14] C. Kassel, Caractère de Chern bivariant, K-Theory 3 (1989), no. 4, 367-400.

[15] A. Kaygun, Bialgebra cyclic homology with coefficients, K-Theory 34 (2005), no. 2, 151-194.

[16] A. Kaygun and M. Khalkhali, Bivariant Hopf cyclic cohomology, preprint, arXiv:math/0606341 [math.KT].

[17] M. Khalkhali and B. Rangipour, Cup products in Hopf-cyclic cohomology, C. R. Math. Acad. Sci. Paris 340 (2005), no. 1, 9-14. 
[18] J.-L. Loday, Cyclic homology, Die Grundlehren der Mathematischen Wissenschaften 301, Springer-Verlag, New York, second edition, 1998.

[19] S. MacLane, Homology, Die Grundlehren der mathematischen Wissenschaften 114, Academic Press Inc., New York, 1963.

[20] I. Nikonov and G.I. Sharygin, Pairings in Hopf-cyclic cohomology of algebras and coalgebras with coefficients, preprint, arXiv:math/0610615v1 [math.KT].

[21] V. Nistor, A bivariant Chern-Connes character, Ann. of Math. (2) 138 (1993), no. 3, 555-590.

[22] D. Quillen, Bivariant cyclic cohomology and models for cyclic homology types, J. Pure Appl. Algebra 101 (1995), no. 1, 1-33.

[23] N. Yoneda, On the homology theory of modules, J. Fac. Sci. Univ. Tokyo Sect. I 7 (1954), 193-227.

Atabey Kaygun kaygun@math.ohio-state.edu

Department of Mathematics, The Ohio State University, Columbus, Ohio, 43210, USA 